\title{
Psychiatry as a therapeutic discipline
}

\author{
W. Gaebel · J. Zielasek
}

(C) Springer-Verlag 2009

Psychiatric therapy is comprised of three basic pillars: pharmacotherapy, psychotherapy, and sociotherapy. Much research has been invested in these fields in the recent years, and impressive progress has been made. The pathophysiology of many mental disorders is becoming better known: the interaction of genes, the environment and learning experiences on the course and development of mental disorders appears to be of central importance. Highly sophisticated research methods such as magnetic resonance tomography techniques are increasing our knowledge on a nearly daily basis. New drugs are constantly being developed, psychotherapy research has turned to evidence-based approaches, and sociotherapy has gained new momentum especially in rehabilitation medicine with the development of novel approaches such as the "supported employment" method. Completely new approaches such as immunization strategies in Alzheimer's disease and deep brain stimulation in affective disorders are being investigated. An integral part of psychiatric therapy is also the question of how best to provide therapy to patients with mental disorders. How should mental healthcare services be structured and organized in order to reach all those in need and provide adequate care? How can the quality of mental healthcare be optimized? Therefore, mental healthcare research is an important issue in any discussion about improving the implementation of therapeutic progress and providing mental healthcare in adequate quantities, with an optimal quality, and at an affordable price. This appears not to be an easy task.

Considering the importance of these questions for our profession and our patients, the central topic of the annual

W. Gaebel $(\bowtie) \cdot$ J. Zielasek

LVR-Klinikum Düsseldorf, Heinrich-Heine-University,

Bergische Landstr 2., 40629 Düsseldorf, Germany

e-mail: wolfgang.gaebel@uni-duesseldorf.de

J. Zielasek

e-mail: Juergen.Zielasek@1vr.de congress of the German Association of Psychiatry and Psychotherapy (Deutsche Gesellschaft für Psychiatrie, Psychotherapie und Nervenheilkunde, hence DGPPN) held in Berlin in November 2008 accordingly became "Psychiatry as a Therapeutic Discipline" following the emphasis on diagnostics in 2007. It was the intent of the DGPPN to assemble a series of invited contributions based on the presentations during the congress to highlight some aspects of Psychiatry as a Therapeutic Discipline. The contributions range from neurobiological research to clinical research and healthcare issues. We hope to provide a supplement issue that may prove helpful to highlight some general and some special aspects of the therapy of mental disorders. Although this conference focused on German contributions, the DGPPN congress gains an increasingly international audience and we accordingly also selected some of the international contributions. Due to space constraints, the supplement issue cannot reflect the state-of-the-art in all areas of mental disorders, but rather reflects a somewhat subjective selection by the Guest Editors. We apologize to many contributors to the DGPPN Congress 2008 whose topics could not be represented in this supplement.

The guest editors wish to thank the Editor-in-Chief of the European Archives of Psychiatry and Clinical Neuroscience, H.J. Möller, for his permission to assemble this supplement issue. We hope that the articles assembled here will advance the scientific discussions about "Psychiatry as a Therapeutic Discipline".

W. Gaebel

President (2007-2008)

German Association of Psychiatry and Psychotherapy

J. Zielasek

Secretary to the Board (2007-2008)

German Association of Psychiatry and Psychotherapy 